${ }^{1}$ Facultad de Medicina Universidad Diego Portales. Santiago de Chile.

Servicio de Anatomía

Patológica. Hospital DIPRECA. Santiago de Chile. anterno.

Fuente de apoyo financiero: Ninguna.

Recibido el 12 de julio de 2010, aceptado el 16 de mayo de 2011

Correspondencia a: Cecilia Varela U Dirección: Ejército 141 Santiago centro, Chile. Fax: (02) 6762901 E-mail: cecyvarela@gmail. com

\section{Hemangiopericitoma de aurícula izquierda. Caso clínico}

\author{
DANIEL BEDMAR M. ${ }^{1, a}$, CECILIA VARELA U. ${ }^{1, \mathrm{a}}$, GINA SQUELLA B. ${ }^{1, \mathrm{a}}$, \\ JOSÉ BELLETTI B. ${ }^{2}$, MARÍA VICTORIA DONOSO ${ }^{1}$

\section{Primary left atrial hemangiopericytoma. Report of one case}

We report a 41-year-old male presenting with progressive dyspnea lasting one month. A CAT scan disclosed a left atrial mass, that was surgically excised. The pathological study of the surgical piece showed a primary hemangiopericytoma. One month later, the patient consulted for cervical pain and a positron emission tomography showed multiple metastases. The patient died two months later.

(Rev Med Chile 2011; 139: 770-773).

Key words: Heart neoplasms; Hemangioma; Hemangiopericytoma.
L os tumores cardiacos constituyen un pequeño grupo dentro de la patología tumoral. El hemangiopericitoma cardiaco es un tumor poco frecuente existiendo escasos reportes en la literatura internacional ${ }^{1-3}$.

El hemangiopericitoma es un tumor vascular originado de los pericitos que habitualmente se ubica en la piel, tejido subcutáneo, músculo de las extremidades, retroperitoneo y cavidades paranasales. Es un tumor de bajo grado de malignidad, sin embargo, con frecuencia recidiva localmente y no responde a adyuvancia con quimioterapia o radioterapia. Por lo tanto, tradicionalmente su tratamiento ha sido quirúrgico ${ }^{4}$.

Se presenta un caso clínico de hemangiopericitoma auricular izquierdo dado lo inusual de esta patología.

\section{Caso clínico}

Paciente de 41 años, sin antecedentes mórbidos de importancia, que consultó por cuadro de un mes de evolución, caracterizado por disnea de rápida progresión, asociado a tos con expectoración hemoptoica y compromiso del estado general. Al ingreso se realizó un AngioTAC que evidenció una masa tumoral, intracavitaria en aurícula izquierda infiltrativa a venas pulmonares (Figura 1).
Se complementó con ecocardiograma confirmando masa hiperdensa de 43 × $38 \mathrm{~mm}$ ocupando el 75\% de la aurícula izquierda (Figura 2).

Se resecó quirúrgicamente cerca de 95\% del tumor por infiltración a venas pulmonares (Figura 3).

El examen histológico demostró la presencia de un tumor fusado con algunas células ovaladas, pericitos, que se disponían en un patrón sólido, interrumpido por espacios vasculares delgados, revestidos por células endoteliales y aplanadas (Figura 4).

El estudio con inmuno-histoquímica demostró reactividad positiva para vimentina en las células proliferantes, CD-34 positivo en el endotelio de los vasos y pericitos (Figura 4), factor VIII positivo sólo en endotelio, así como negatividad para S-100 y CD-68. Estos hallazgos, junto con el claro patrón de reticulina en las técnicas argénticas, indicaron el carácter pericítico de la neoplasia y se confirmó su carácter benigno histológico, con un índice mitótico inferior a tres mitosis por 10 campos de mayor aumento.

El paciente evolucionó satisfactoriamente post cirugía y se decidió el alta.

Al mes siguiente, volvió a consultar por presentar dolor cervical intenso asociado a odinofagia. Se realizó estudio de diseminación con PET-CP 


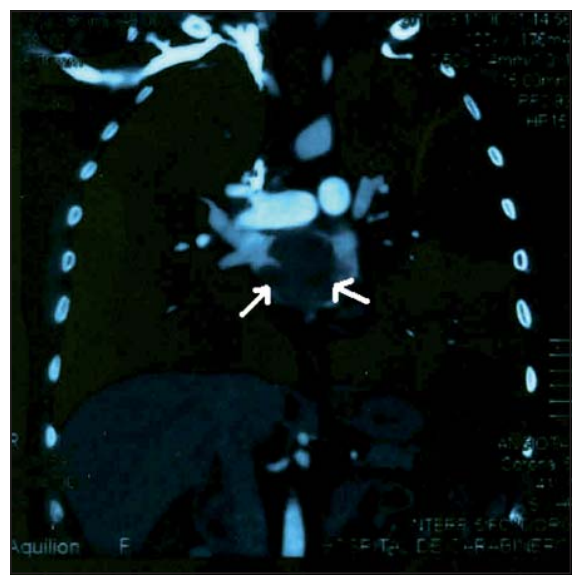

Figura 1. AngioTAC evidencia masa intracavitaria en aurícula izquierda de $40 \times 40 \mathrm{~mm}$, infiltrativa a venas pulmonares.

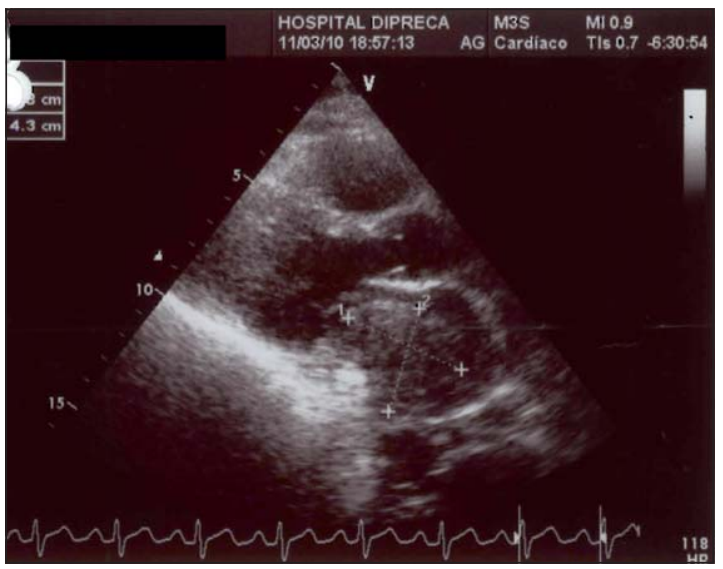

Figura 2. Ecocardiograma 2D TT que revela: $A l 45 \mathrm{~mm}, \mathrm{VI}$ $43 / 30 \mathrm{MM}, \mathrm{FEVI} 60 \%$, masa hiperdensa de $43 \times 38 \mathrm{~mm}$, que ocupa el $75 \%$ de la cavidad Al, ocasionando un gradiente transluminal medio $31 \mathrm{~mm}$ y una reducción del área valvular mitral funcional a $0,8 \mathrm{~cm}^{2}$

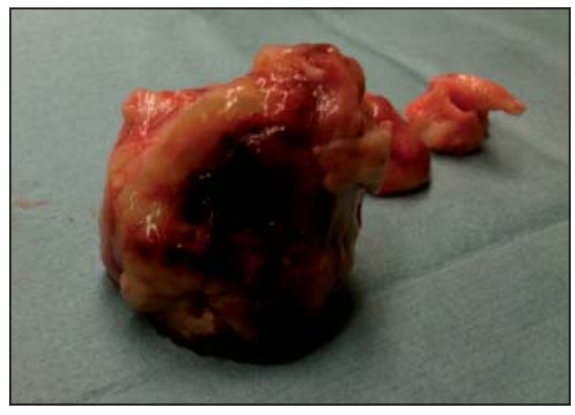

Figura 3. Examen macroscópico: formación tumoral, mamelonada, blanquecino grisácea, con áreas pardo rojiza, consistencia elástica de $5,5 \times 5 \mathrm{~cm}$. Dos formaciones nodulares de $4 \times 4 \mathrm{~cm}$, y algunos fragmentos laminares $2 \times 1,5 \mathrm{~cm}$ de iguales características. que mostraba zonas hipermetabólicas en pulmón, base de cráneo, hígado, amígdala izquierda, aurícula izquierda, suprarrenal izquierda, cola de páncreas y colon descendente. Esto se explicó por embolización del tumor a la circulación sistémica.
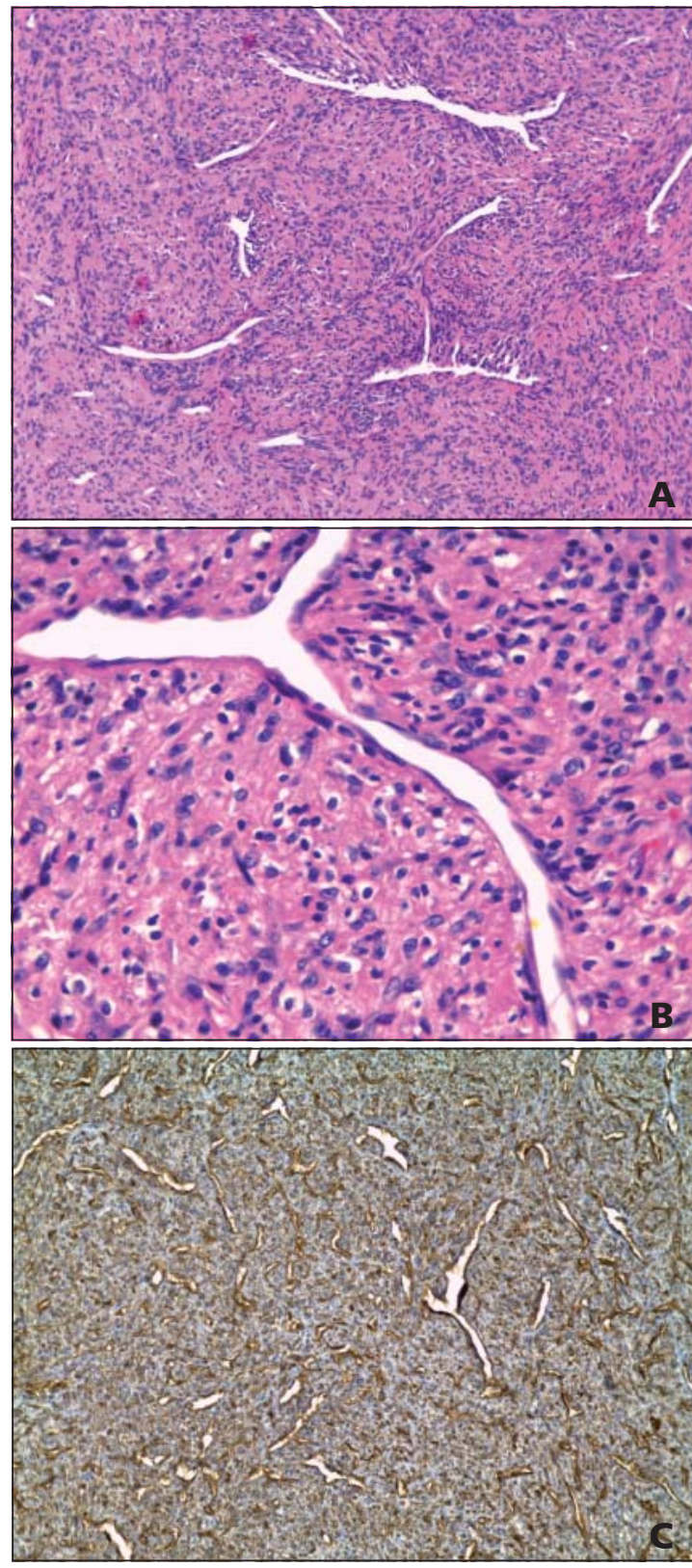

Figura 4. Tinción Hematoxilina-Eosina (HE), Muestra proliferación celular sólida y formaciones vasculares con ramificaciones en "asta de ciervo" típicas del hemangiopericitoma. Se realizó inmunohistoquímica siendo positivo el CD34. A: H\&E X 10. B: H\&E x 40. C: Cd 34. 
Se presentó caso a comité oncológico que decidió manejo paliativo y del dolor. A los 2 meses, el paciente falleció de falla multiorgánica.

\section{Discusión}

La patología tumoral primaria del corazón constituye entre $0,0017 \%$ y $0,05 \%$ de todos los tumores malignos, siendo lo más frecuente las metástasis ${ }^{5}$.

Actualmente existen pocos casos publicados en la literatura de hemangiopericitoma primario cardiaco. Todos los reportes corresponden a tumores auriculares. Además presenta características clínicas y morfológicas indistinguibles con el mixoma auricular, siendo uno de los principales diagnósticos diferenciales ${ }^{1-3}$. El hemangiopericitoma es una neoplasia mesenquimática inusual, que deriva de los pericitos vasculares de Zimmermann. Fue descrito originalmente por Stout y Murray en $1942^{6-8}$. Corresponde al 1\% de todos los tumores vasculares ${ }^{2}$, apareciendo con mayor frecuencia en edad adulta, y sólo en 5-10\% de los casos ocurre en niños ${ }^{7}$, sin tener predilección por sexo ${ }^{7,9}$. Este tumor está compuesto por células fusiformes llamadas pericitos que forman una red de espirales que rodean a canales vasculares prominentes ${ }^{6-8}$.

La inmuno-histoquímica del hemangiopericitoma muestra respuesta a vimentina, colágeno tipo IV y respuesta negativa al antígeno VIII, proteína S-100, enolasa neuro específica, antígeno carcinoembrionario, desminas, laminina y citoqueratina $^{10}$.

La ubicación más frecuente de estos tumores son la piel, tejido subcutáneo, músculo de las extremidades y retroperitoneo. Sin embargo, es raro encontrarlo en corazón o en mediastino ${ }^{11}$.

El hemangiopericitoma ubicado en aurícula izquierda tiene un cuadro clínico caracterizado por disnea progresiva que luego se hace severa asociado a compromiso hemodinámico en etapas más avanzadas ${ }^{2}$.

El tratamiento de elección es la extirpación quirúrgica. Se ha recomendado quimioterapia o radioterapia post cirugía, pero en varios estudios resultó ser inefectiva ${ }^{12}$. Por otro lado, hay autores que recomiendan esta terapia adyuvante, sin embargo, actualmente este tema sigue en controversia.

Aproximadamente $50 \%$ de los hemangiopericitomas recurren dentro de los primeros 5 años del diagnóstico ${ }^{9,10}$. Las recurrencias son frecuentemente encontradas en tórax, pulmón, pleura, hígado, cerebro y hueso ${ }^{13,14}$.

El diagnóstico diferencial se hace con tumores que exhiben similitud histológica tales como el glomangioma, sarcoma hemangioendotelial, leiomioma vascular, leiomioblastoma ${ }^{15}$, histiocitoma fibroso y condrosarcoma mesenquimal, entre otros. Recientemente, el tumor fibroso solitario, ha sido reconocido como principal diagnóstico diferencial del hemangiopericitoma dada su gran similitud histológica. La gran diferencia entre ambos tumores radica en el mejor pronóstico del tumor fibroso solitario post resección quirúrgica y rara vez metastiza ${ }^{16}$.

\section{Referencias}

1. Orlandi A, Ferlosio A, Ciucci A, Pellegrino A, Spagnoli L. Unusual cardiac tumour with perivascular mixoide differentiation: a case report. Clin Pathol 2004; 57: 133840.

2. Ozaki N, Mukohara N, Yoshida M, Shida T. Successful resection of giant hemangiopericytoma originating from the left atrium. Interac Cardiovascular Thorax Surg 2006; 5: 79-80.

3. Ghiribelli C, Campione A, Ligabue T, Di Bisceglie M, Severi S, Gentilini R. A case of primary malignant hemangiopericytoma of the heart with fatal outcome. J Cardiovasc Sur. 2006; 47 (1): 71-3.

4. Schiariti M, Goetz P, El-Maghraby H, Tailor J, Kitchen N. Hemangiopericytoma long-term outcome revisited. J Neurosurg 2011; 114 (3): 747-55.

5. Becker P, Ramírez A, Zalaquett R, Morán S, Irarrázaval M, Arretz C, et al. Mixoma cardiaco: Caracterización clínica, métodos diagnósticos y resultados alejados del tratamiento quirúrgico durante tres décadas de experiencia. Rev Med Chile 2008; 136: 287-95.

6. Hart LL, Weinberg JB. Metastatic hemangiopericytoma with prolonged survival. Cancer. 1987; 60: 916-920.

7. Hervé S, Abd Alsamad I, Beautru R, Gaston A, Bedbeder $\mathrm{P}$, Peynègre R, et al. Management of sinonasal hemangiopericytomas. Rhinology 1999; 37: 153-8.

8. Rodríguez C, Ramsey K, Jenkins J, Poquette C, Kaste S, Merchant T, et al. Hemangiopericytoma in children and infants. Cancer 2000; 88: 198-204.

9. Catalano P, Brandwein M, Shah D, Urken M, Lawson W, Biller H. Sinonasal hemangiopericytomas: a clinicopathologic and immunihistochemical study of seven cases. Head Neck 1996; 18: 42-53.

10. Espat J, Lewis J, Leung D, Woodruff M, Antonescu C, 
Jingu S, et al. Conventional hemangiopericytoma: Modern analysis of outcome. Cancer. 2002; 95: 1746-51.

11. Kanazawa T, Nishino H, Miyata M, Kuriki K, Abe K, Ichimura K. Hemangiopericytoma of infratemporal fossa. J Laryngol Otol 2001; 115: 77-9.

12. Aziz Z, Zahid M, Qazi S, Zaman H. Haemangiopericytoma of the heart: report of a case with combined modality treatment. Eur J Surg Oncol 1997; 23 (5): 459-61.

13. Yoshida M, Morita M, Kakimoto S, Kawakami M, Sasaki S. Primary hemangiopericytoma of the trachea. Ann Thorac Surg 2003; 76: 944-6.

14. Gómez M, Paya L, Parede J, Aranda L, Massuti B, Ta- lavera J. Hemangiopericytoma of the soft palate and mediastinum: a case report. Acta Otorrinolaringol Esp 1994; 45: 465-8.

15. Hahn M, Dawson R, Esterly J, Joseph D. Hemangiopericytoma: An ultraestructural study. Cancer 1973; 31: 225-61.

16. Yutaka H, Naoyuki U, Yasuhiko H, Mitsutoshi N, Masayuki I, Daisuke K, et al. A reevaluation of the primary diagnosis of hemangiopericytoma and the clinical importance of differential diagnosis from solitary fibrous tumor of the central nervous system. Clinical Neurology and Neurosurgery 2009; (111): 34-8. 